\title{
Numerical Simulation of Transpiration Cooling for a High Speed Vehicle with Substructure
}

\author{
Imran Naved *, Tobias Hermann ${ }^{\dagger}$ and Matthew McGilvray ${ }^{\ddagger}$ \\ The University of Oxford, Department of Engineering Science, Southwell Building, Osney Mead, Oxford OX2 OES, \\ United Kingdom
}

This paper presents a numerical model which assesses the effect of applying transpiration cooling to both the outer wall and the substructure of a high speed flight vehicle. The PIRATE transpiration cooling code has been extended and validated to account for quasitwo-dimensional lateral heat conduction effects, thereby allowing for analysis of more complex geometries. This enables very fast calculations of the two-dimensional transient temperature response of a transpiration cooled thermal protection system, suitable for first order systems studies. To solve for the transpiration cooled outer wall and two-dimensional solid substructure, PIRATE has been coupled with the commercial finite element package COMSOL. This enables modelling of the longer duration thermal effects of the integrated heat load over a flight trajectory. Transpiration cooling using Helium coolant has been applied to a wing leading edge model with an aluminium substructure. Carbon-Carbon cermaic composite (C/C) and the ultra high temperature ceramic (UHTC) $\mathrm{ZrB}_{2}$ are chosen as candidate materials. Results for the substructure temperature history for the Space Shuttle re-entry trajectory are obtained showing that transpiration cooling can lead to a $35 \%$ reduction in peak substructure temperature and a $65 \%$ reduction in thermal gradients.

\section{Nomenclature}

A Area, $\mathrm{m}^{2}$

$c_{p} \quad$ Specific heat capacity, $\mathrm{J} \mathrm{kg}^{-1} \mathrm{~K}^{-1}$

$F \quad$ Blowing ratio, $\left(\rho_{f} u_{f}\right) /\left(\rho_{g} u_{g}\right)$

$h_{v} \quad$ Volumetric heat transfer coefficient, $\mathrm{W} \mathrm{m}^{-3} \mathrm{~K}^{-1}$

$k \quad$ Thermal conductivity, $\mathrm{W} \mathrm{m}^{-1} \mathrm{~K}^{-1}$

$K_{D} \quad$ Darcy coefficient, $\mathrm{m}^{2}$

$K_{F} \quad$ Forchheimer coefficient, $\mathrm{m}$

$L \quad$ Thickness, $\mathrm{m}$

*DPhil student, Hypersonics group, Oxford Thermofluids Institute, AIAA Member.

${ }^{\dagger}$ Senior Research Associate, Hypersonics group, Oxford Thermofluids Institute, AIAA Member.

$¥$ Associate Professor, Hypersonics group, Oxford Thermofluids Institute, AIAA Member. 


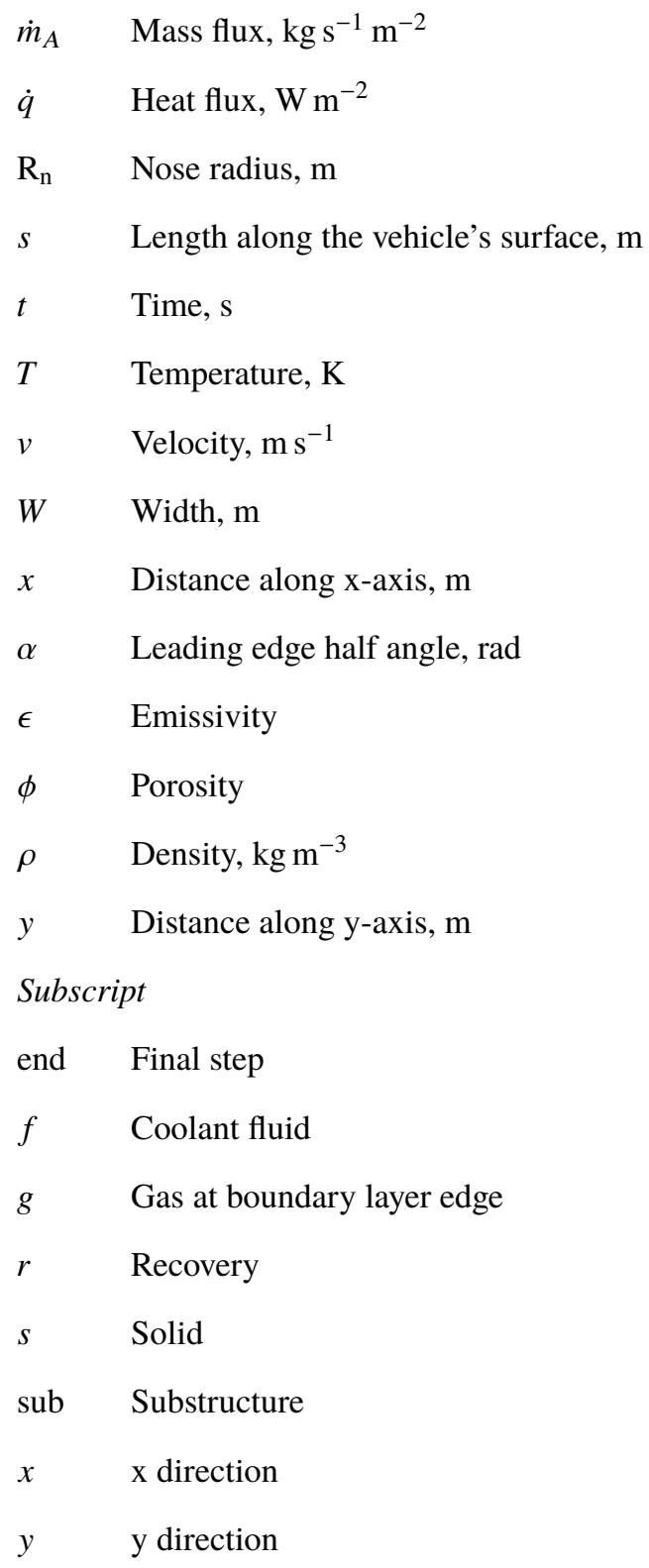

\section{Introduction}

Hypersonic vehicles are exposed to very high heat fluxes and heat loads during re-entry or sustained flight. This requires thermal protection systems to manage these heat fluxes and keep wall temperatures below critical values. Transpiration cooling is an active cooling techology that can enable fully re-usable hypersonic vehicles. It relies on a gaseous or liquid fluid which is fed through a porous wall [1]. The coolant leads to internal convective cooling between the porous solid and the fluid, and a protective film that keeps the hot external gas away from the surface [2]. Transpiration cooling provides alternative flexibility in the design of future re-usable hypersonic vehicles. 
Only a few systems level studies of transpiration cooling have been performed. Cowell and Modlin [3] employed a finite difference code to investigate the feasibility of cooling hypersonic wing leading edge structures using metal heat pipe and surface mass transfer cooling techniques, including transpiration cooling. Modlin and Cowell [4] then applied this code to the transient thermal behaviour of cooling SCRAMJET inlets using the aforementioned hypersonic leading-edge cooling model, modified to include type IV shock interaction surface heating effects [5, 6]. It was found that a combination of transpiration, film and heat pipe cooling techniques can keep surface temperatures at acceptable levels. Böhrk et al [7] developed the finite difference code HEATS to determine the transient wall heat flux to a transpiration cooled flat plate under laminar and turbulent conditions. Both of these models considered an adiabatic backside and did not consider the effects of the integrated heat load to the substructure of the vehicle. Hermann et al. [8] applied the one-dimensional PIRATE numerical code to conduct systems studies of transpiration cooling for a blunt cone. This analysis considered radiation coupling between the transpiration cooled skin and the substructure, but assumed that the substructure is at constant temperature. Steelant et al [9] applied a constant heat flux to a finite element model of an uncooled SHEFEX II TPS panel showing that substructure temperatures can rise well after the outer TPS reaches radiative equilibrium, and potentially violate structural limits.

This paper investigates how transpiration cooling can be applied to wing leading edges to lower the backside temperatures of the porous wall and therefore the integrated heat load to the substructure. To achieve this, firstly, the Oxford in-house PIRATE code is extended to model the two-dimensional effects of lateral conduction within the porous outer wall. Secondly, the PIRATE code is coupled to the commercial finite element package COMSOL to model the thermal behaviour of the substructure during a particular flight trajectory. The resulting coupled methodology is applied to a $0.1 \mathrm{~m}$ nose radius, $15^{\circ}$ half angle wing leading edge with the Space Shuttle re-entry trajectory. The results show a significant substructure temperature reduction due to the application of cooling as well as reduced thermal gradients.

\section{One-Dimensional PIRATE model}

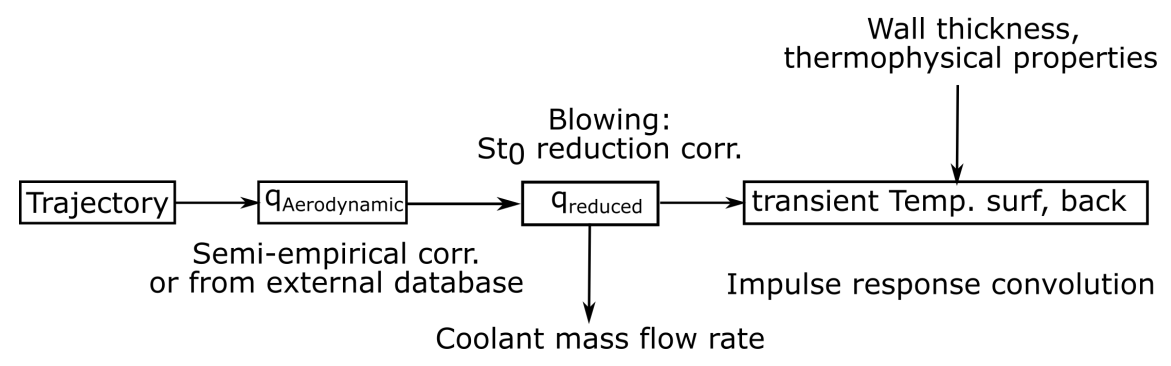

Fig. 1 Flow chart of the computational approach $[\mathbf{8}]$.

The numerical code Porous Impulse Response Analyis for Transpiration cooling Evaluation (PIRATE) [8] calculates the transient temperature response for a one-dimensional porous material subjected to aerodynamic surface heating. 
By relying on a method based on impulse response convolution, significantly faster computation times are possible providing the opportunity to conduct optimisation studies for various flight trajectories and geometries. Figure 1 shows an outline of the code. For a given trajectory, the aerodynamic heat fluxes with no cooling are calculated from aerodynamic correlations or imported from an external database. The cooling effect of the local heat flux mitigation through blowing and downstream film effectiveness is then taken into account using semi-empirical models [10, 11].

The cooling within the inner structure of a porous material is carried out by convective heat transfer between the material and the coolant. Therefore, the one dimensional governing equations for the solid and fluid phases are [12]

$$
(1-\phi) \rho_{s} c_{p, s} \frac{\partial T_{s}}{\partial t}=k_{y, s}(1-\phi) \frac{\partial^{2} T_{s}}{\partial y^{2}}+h_{v}\left(T_{f}-T_{s}\right)
$$

and

$$
\phi \rho_{f} c_{p, f} \frac{\partial T_{f}}{\partial t}=\phi \rho_{f} c_{p, f} \frac{v_{f}}{\phi} \frac{\partial T_{f}}{\partial y}+h_{v}\left(T_{s}-T_{f}\right),
$$

where (s) denotes the solid phase, (f) the fluid phase, $k$ the thermal conductivity, $\rho$ the density, $T$ the temperature, $c_{p}$ the specific heat capacity, $\phi$ the porosity, $h_{v}$ the volumetric heat transfer coefficient, $t$ the time, and $y$ the location medially through the material.

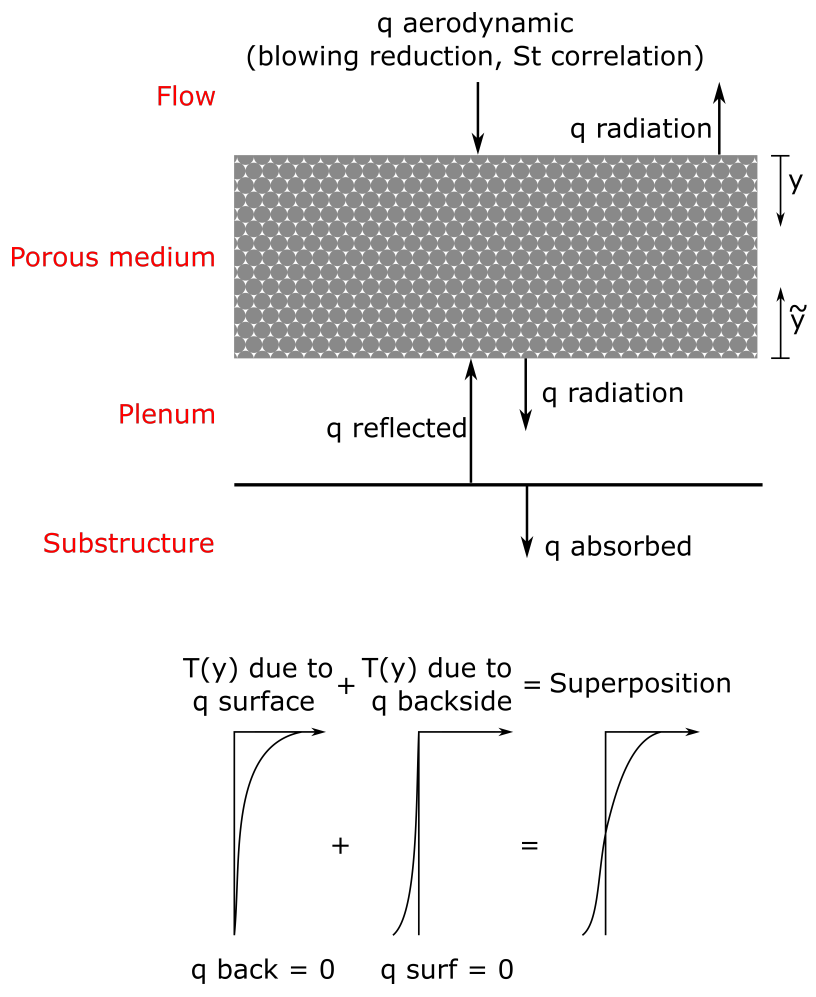

Fig. 2 Schematic of the one-dimensional thermal model [8].

Figure 2 shows a schematic of the one-dimensional thermal model. The temperature of the solid and fluid phases 
are calculated based on a given coolant mass flow rate, porous wall thickness and the thermophysical properties of the solid and fluid phases. The transient temperature calculations are based on an impulse response approach which solves the coupled fluid-solid heat conduction problem with radiation cooling to a constant temperature substructure. As the equations are solved analytically for the normal direction, constant material properties are assumed. This is an inherent limitation of this numerical method and is acceptable given the usage of this numerical code for first order system studies. In addition, it should be noted that the faster computation times are achieved by calculating the surface and backside temperatures directly, the temperature at different intermediate locations through the material are not available without additional computations. This approach is detailed further in Hermann et al [8].

The PIRATE model has been validated against a variety of experiments, both ground based and re-entry flight testing. In particular, experimental results from the SHEFEX II flight test campaign, and the RESPACE and IMENS+ campaigns are used [13, 14]. The different validation cases have demonstrated that this numerical model matches experimental data closely and agrees with experimental measurements to within $10 \%$. The relevant effects occurring during transpiration cooled hypersonic flight, i.e. transient heating, local cooling through blowing, and downstream film coverage, are all replicated within acceptable accuracy.

\section{Extension of PIRATE to quasi-two-dimensional heat transfer}

A key assumption made in PIRATE is that only one-dimensional heat conduction and coolant flow through the porous material is considered. Therefore, when a calculation is conducted across a full body, a series of one-dimensional models are solved independently. Solving for full two-dimensional material conduction and coolant flow is a complex problem that requires knowledge of the complete temperature distribution inside the material. An intermediate solution, suitable for the purposes of rapid system studies, is to only consider two-dimensional conduction and assume coolant flow to be solely in the normal direction. This is a reasonable assumption for many geometries where lateral pressure gradients are small i.e. the radius of curvature of the surface is much larger than the thickness of the material. Thus, the governing equations for the solid and fluid phases including two-dimensional conduction are:

$$
\underbrace{(1-\phi) \rho_{s} c_{p, s} \frac{\partial T_{s}}{\partial t}}_{\text {Transient heating (solid) }}=\underbrace{k_{x, s}(1-\phi) \frac{\partial^{2} T_{s}}{\partial x^{2}}}_{\text {Lateral conduction (solid) }}+\underbrace{k_{y, s}(1-\phi) \frac{\partial^{2} T_{s}}{\partial y^{2}}}_{\text {Normal conduction (solid) }}+\underbrace{h_{v}\left(T_{f}-T_{s}\right)}_{\text {Fluid-solid heat exchange }}
$$

and

$$
\underbrace{\phi \rho_{f} c_{p, f} \frac{\partial T_{f}}{\partial t}}_{\text {Transient heating (fluid) }}=\underbrace{\phi \rho_{f} c_{p, f} \frac{v_{f}}{\phi} \frac{\partial T_{f}}{\partial y}}_{\text {Convection }}+\underbrace{h_{v}\left(T_{s}-T_{f}\right)}_{\text {Solid-fluid heat exchange }}
$$

By considering only the lateral conduction term in $\mathrm{Eq} 3$, the corresponding governing equation for purely onedimensional transient conduction in the $x$ (lateral) direction ignoring any convective heat transfer between the solid and 
fluid phases is:

$$
\frac{\partial T_{s}}{\partial t}=\frac{k_{x, s}}{\rho_{s} c_{p, s}} \frac{\partial^{2} T_{s}}{\partial x^{2}}
$$

where $T_{s}(x, t)$ is the temperature of the solid material at spatial point $x$ and time $t$.

To model lateral conduction, a quasi two dimensional approach is followed where conduction medially through the material is calculated first using the impulse response method of PIRATE detailed above. Thereafter, the CrankNicholson scheme for one dimensional thermal conduction is applied at every time step to the existing surface and backside temperature history. The advantage of using Crank-Nicholson is its known unconditional stability for both linear and non-linear heat conduction systems [15]. The Crank-Nicholson scheme can be expressed as:

$$
\frac{T_{x}^{t}-T_{x}^{t-1}}{\Delta t}=\frac{\alpha}{2}\left[\frac{T_{x-1}^{t}-2 T_{x}^{t}+T_{x+1}^{t}}{\Delta x^{2}}+\frac{T_{x-1}^{t-1}-2 T_{x}^{t-1}+T_{x+1}^{t-1}}{\Delta x^{2}}\right]
$$

where $x$ is the spatial step along the $\mathrm{x}$ axis (from $x=0$ to $\mathrm{W}$ ), $t$ is the time step, $\Delta t$ is the time step discretisation, $\Delta x$ is the spatial discretisation and $\alpha$ is the thermal diffusivity defined as $\frac{k_{x, s}}{\rho_{s} c_{p, s}}$. Neumann boundary conditions are considered such that there is zero heat transfer across the sides (adiabatic boundary condition),

$$
\left.\frac{\partial T}{\partial x}\right|_{x=0, \mathrm{~W}}=0
$$

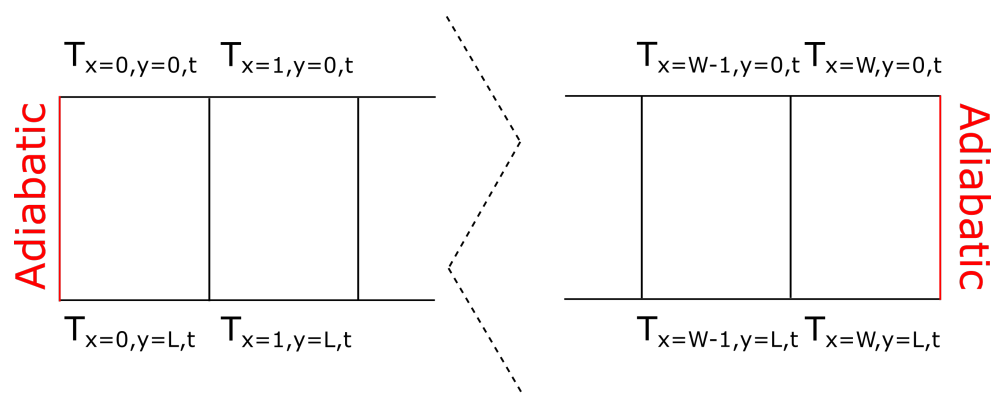

Fig. 3 PIRATE 1D output tempertature field.

This scheme is applied to the existing transient temperature distribution from PIRATE via the following steps:

1) The PIRATE 1D simulation is applied to each node. As per Fig. 3 this results in a temperature field $T_{x, y, t}$ describing every node of the two-dimensional material $(x, y)$ at each time step $t$.

2) An array of differential temperatures $\left(d T_{x, y, t}=T_{x, y, t+1}-T_{x, y, t}\right.$, ) for the surface and backside are calculated between each time step. These differential temperatures represent an instantaneous temperature rise (or fall) due to the heat flux applied in each time-interval $d t$. As these are based on the 1D conduction model, they have to be 
relaxed through lateral conduction, i.e. the heat will spill over to neighbouring nodes.

3) The Crank-Nicholson algorithm is applied to the differential temperatures at the surface and backside separately. This results in a $t$ by $\tilde{t}$ matrix where the 1D heat conduction equation is solved in the lateral direction for every spatial node from $x=0-\mathrm{W}$ at $y=0$ (the surface) and $y=\mathrm{L}$ (backside):

$$
d T_{x, y, t, \tilde{t}}^{\mathrm{lat}}=\left(\begin{array}{cccc}
d T_{x, y, t_{1}, \tilde{t}_{1}}^{\mathrm{lat}} & d T_{x, y, t_{1}, \tilde{t}_{2}}^{\mathrm{lat}} & \cdots & d T_{x, y, t_{1}, \tilde{t_{\text {end }}}}^{\mathrm{lat}} \\
d T_{x, y, t_{2}, \tilde{t}_{1}}^{\mathrm{lat}} & d T_{x, y, t_{2}, \tilde{t}_{2}}^{\mathrm{lat}} & \cdots & d T_{x, y, t_{2}, \tilde{t}_{\text {end }}}^{\mathrm{lat}} \\
\vdots & \vdots & \ddots & \vdots \\
d T_{x, y, t_{\text {end }}, \tilde{t_{1}}}^{\mathrm{lat}} & d T_{x, y, t_{\text {end }}, \tilde{t_{2}}}^{\mathrm{lat}} & \cdots & d T_{x, y, t_{\text {end }}, \tilde{t_{\text {end }}}}^{\mathrm{lat}}
\end{array}\right),
$$

where the new temperature field has values at two distinct times, $t$ and $\tilde{t}$. Both of these range from $0-t_{\text {end }}$ where $t$ represents the transient change in temperature due to one dimensional medial conduction at a given time and $\tilde{t}$ represents the change due to lateral conduction. These two separate time variables occur as essentially two independent conduction problems have been solved - in the normal direction by applying the impulse response method of PIRATE and laterally using the Crank-Nicholson method.

4) The two independent transient conduction solutions are then combined to form the two-dimensional temperature field. This is accomplished by appropriately weighting the contributions of both conduction terms at every time step, i.e for example at time step $t_{2}, T_{x, y, 2}=d T_{x, y, 2,1}+d T_{x, y, 1,2}+T_{x, y, 0}$. This process is displayed in Fig. 4 where a schematic of the quasi $2 \mathrm{D}$ method is shown for an arbitrary temperature distribution. For example at $t_{2}$, the $1 \mathrm{D}$ conduction solution at $t_{2}$ is added to the solution at $t_{1}$ with lateral conduction applied to $\tilde{t}_{2}$. This can also be represented as the summation of a diagonal of the matrix in Eq. 8 where the resulting temperature field is:

$$
T_{x, y, t_{\text {end }}}^{\mathrm{lat}}=T_{x, y, 0}^{\mathrm{lat}}+\sum_{t=1}^{t_{\text {end }}} d T_{x, y, t, t_{\mathrm{end}}+1-t}^{\mathrm{lat}},
$$

where $t_{\text {end }}$ is the time step of interest, $T_{x, y, 0}^{\text {lat }}$ is the initial temperature distribution and $T_{x, y, t_{\text {end }}}^{\text {lat }}$ is the new temperature distribution with lateral conduction applied.

The key advantage of this approach is the very quick computational time. On top of the one-dimensional calculation time, this method solves for the two-dimensional field with only a $20 \%$ increase in computation time . For a typical calculation with 2000 time steps and 60 spatial nodes, a complete trajectory simulation takes only around 60-75 minutes. This enables first order systems studies of the application of transpiration cooling for different trajectories, geometries 


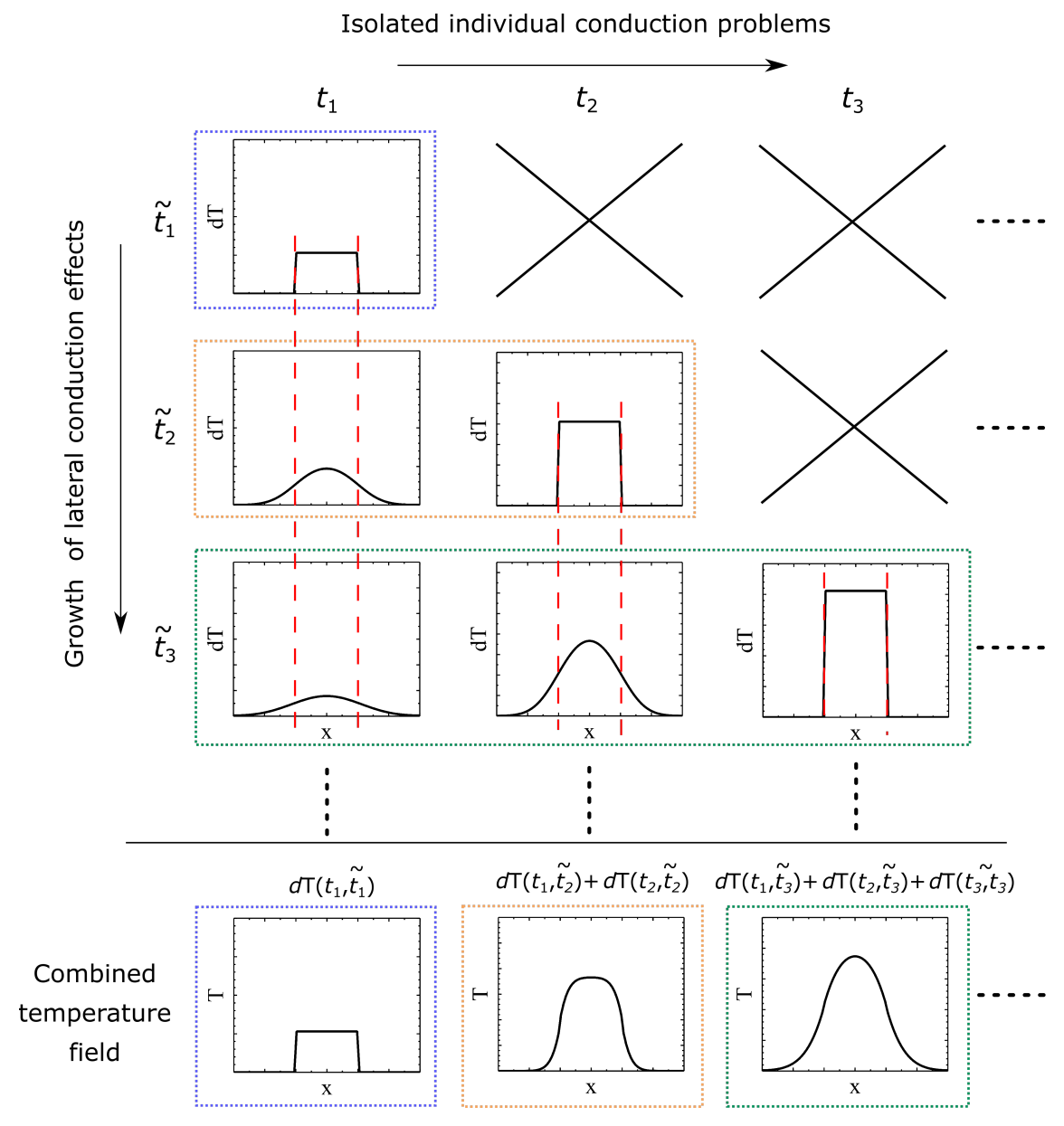

Fig. 4 Schematic of the 2D conduction method.

and materials to be feasible on a desktop computer. Henceforth, the quasi-two-dimensional version of PIRATE will be referred to as 'PIRATE Q2D' whilst the original one-dimensional code is 'PIRATE 1D'.

This method of calculating lateral conduction is applicable for moderate thermal gradients where the effects of variable wall thickness and two-dimensional coolant mass flow do not dominate.

\section{Validation of the quasi two-dimensional heat conduction model}

\section{A. Uncooled - COMSOL Finite Element Analysis}

Firstly, this method to solve for lateral conduction has been validated by comparing the temperature response of the PIRATE Q2D code with the COMSOL commercial finite element package. For zero coolant mass flux, the differential equations for the solid and fluid phases of the porous material reduce to the well known equations for two dimensional conduction. In this case, the temperature response via the quasi two dimensional method described here can be compared directly with a commercial finite element package like COMSOL [16].

Two high temperature porous materials are chosen; the main purpose of which is to confirm that the quasi 


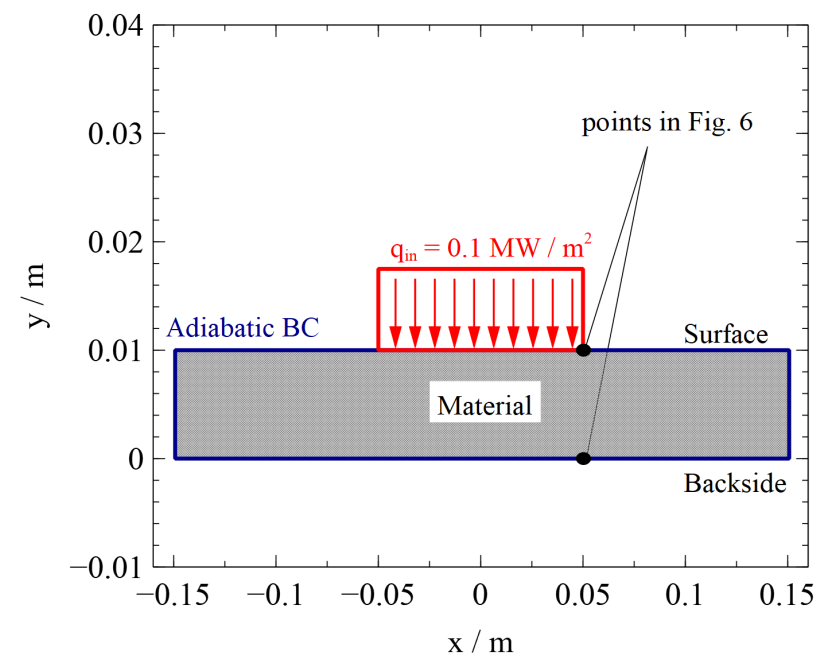

Fig. 5 Schematic of the validation case.

two dimensional approach accurately models the transient change in temperature through the material. These are, Carbon-Carbon (C/C) ceramic composite [1], and the ultra high temperature ceramic (UHTC) ZrB 2 [17]. The material parameters used in this study are summarised in Table 1 Both materials have been investigated in previous transpiration cooling studies for high speed vehicles [2, 13].

\begin{tabular}{ccc}
\hline \hline Material & UHTC [17] & C/C [1,7] \\
\hline$\phi$ & 0.41 & 0.13 \\
$\epsilon$ & 0.7 & 0.85 \\
$k_{x, s} / \mathrm{Wm}^{-1} \mathrm{~K}^{-1}$ & 41.4 & 14 \\
$k_{y, s} / \mathrm{Wm}^{-1} \mathrm{~K}^{-1}$ & 41.4 & 2 \\
$c_{p, s} / \mathrm{J} \mathrm{kg}^{-1} \mathrm{~K}^{-1}$ & 437 & 1650 \\
$\rho_{s} / \mathrm{kg} \mathrm{m}^{-3}$ & 6100 & 1400 \\
$K_{D} / \mathrm{m}^{2}$ & $3.4 \cdot 10^{-14}$ & $1.645 \cdot 10^{-13}$ \\
$K_{F} / \mathrm{m}^{-3}$ & $15.7 \cdot 10^{-8}$ & $2.9 \cdot 10^{-6}$ \\
$h_{v} / \mathrm{W} \mathrm{m} \mathrm{K}^{-1}$ & $5.5 \cdot 10^{4}$ & $1 \cdot 10^{4}$ \\
\hline \hline
\end{tabular}

Table 1 Material properties used in this study. Note: The $K_{D}$ and $K_{F}$ terms for UHTC were measured using the ISO standard rig of Ifti et al.[18] and C/C by applying the pressure decay method of Innocentini et al.[19]

The two dimensional model described in Fig.5 is used as a validation case. Initially the material is set at $300 \mathrm{~K}$. Subsequently, a step change in heat flux of $0.1 \mathrm{MW} \mathrm{m}^{-2}$ is applied at the centre (from $\mathrm{x}=-0.05-0.05 \mathrm{~m}$ ) of the model for $20 \mathrm{~s}$ and then allowed to thermally dissipate until $100 \mathrm{~s}$. Adiabatic boundary conditions are assumed for the remainder of the boundaries. This represents a highly two-dimensional and transient heat transfer case where the front and backside temperatures initially strongly diverge, eventually approaching equilibrium for the steady state. COMSOL solves for heat conduction via the finite element method. For this validation model, the COMSOL mesh has a maximum element 
size of $0.003 \mathrm{~m}$ leading to 891 domain elements and 209 boundary elements. Time stepping is achieved with a relative tolerance of 0.0001 .

Mesh sensitivity analysis has been carried out for the COMSOL simulation. Three meshes were compared: a coarse mesh with 47 elements, a medium mesh with 891 elements and a fine mesh with 84062 elements. Less than $0.01 \%$ difference in the final solution was found between the medium and fine meshes. For this reason, the medium COMSOL mesh was used for all subsequent simulations.

\begin{tabular}{cccc}
\hline \hline $\mathrm{dt} / \mathrm{s}$ & $\Delta \mathrm{T}_{\max } / \mathrm{K}$ & $\Delta \mathrm{T}_{\max } / \%$ & Simulation time / \\
\hline 0.5 & 35.57 & 6.68 & 13 \\
0.25 & 26.81 & 5.02 & 28 \\
0.1 & 18.11 & 3.39 & 86 \\
0.05 & 13.26 & 2.48 & 280 \\
0.025 & 9.60 & 1.79 & 1286 \\
\hline
\end{tabular}

Table 2 Time step sensitivity study. Note: $\Delta \mathrm{T}_{\max }$ is the maximum temperature difference between PIRATE Q2D and COMSOL. This is recorded in both absolute and percentage terms.

\begin{tabular}{cccc}
\hline \hline $\mathrm{dx} / \mathrm{m}$ & $\Delta \mathrm{T}_{\max } / \mathrm{K}$ & $\Delta \mathrm{T}_{\max } / \%$ & Simulation time / s \\
\hline 0.02 & 64.79 & 16.14 & 1658 \\
0.01 & 31.38 & 7.82 & 3002 \\
0.005 & 12.66 & 3.15 & 4710 \\
0.0025 & 4.59 & 1.14 & 11196 \\
\hline \hline
\end{tabular}

Table 3 Spatial discretisation sensitivity study with $\mathrm{dt}=0.05 \mathrm{~s}$.

The advantage of the impulse response method is that the temperature distribution vertically through the material can be solved analytically with the accuracy of the final solution only dependant on the time step, dt. Firstly, a time step sensitivity study is conducted by setting up a PIRATE 1D simulation (no lateral conduction effects considered) at the centre of the validation model $(\mathrm{x}=0 \mathrm{~m})$ with the $\mathrm{C} / \mathrm{C}$ material. The transient surface temperature evolution of COMSOL and PIRATE 1D, with several different time steps from $\mathrm{dt}=0.025$ to $\mathrm{dt}=0.5 \mathrm{~s}$, are shown in Fig. 6a. For each time step, the time taken to complete each simulation and the peak temperature difference between PIRATE 1D and COMSOL is shown in Table 2, where the simulations are completed using a 6 core, 3.2 Ghz desktop computer with 64 GB RAM. To achieve numerical accuracy whilst ensuring quick calculation times, $\mathrm{dt}=0.05 \mathrm{~s}$ (highlighted in cyan) is chosen for all subsequent simulations. For this time step, there is a peak temperature difference of $2.48 \%$ (or $13.26 \mathrm{~K}$ ) compared to COMSOL with a simulation time of $280 \mathrm{~s}$.

In addition, a similar study is conducted to investigate the sensitivity of temperature distributions to spatial discretisation in the $\mathrm{x}$ direction, $\mathrm{dx}$. This is important for the validation case due to the very large spatial thermal 


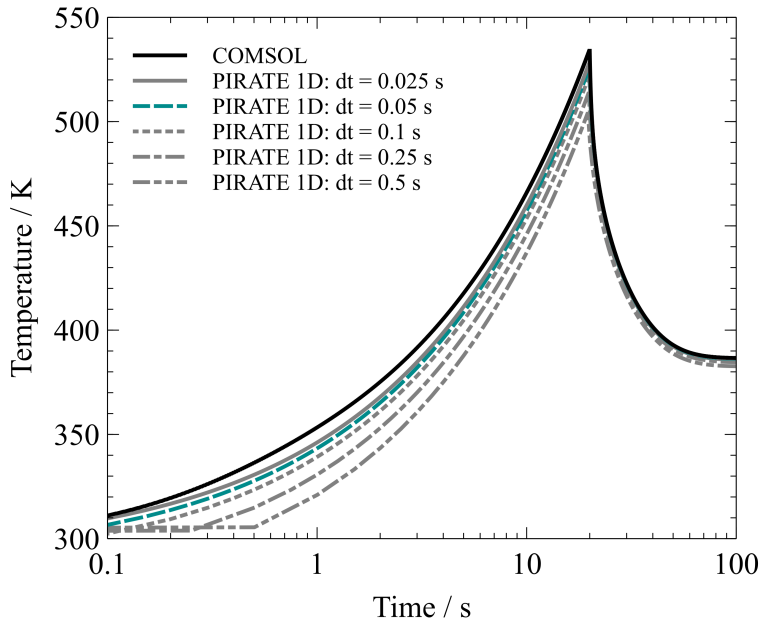

(a) Time discretisation, at $\mathbf{x}=0 \mathrm{~m}$

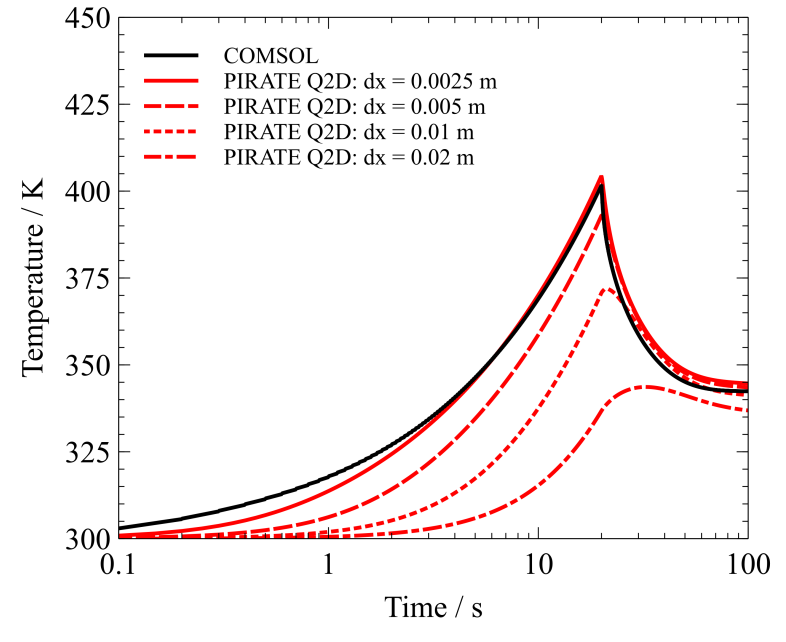

(b) Spatial discretisation, assuming $\mathrm{dt}=0.05 \mathrm{~s}$ at $\mathrm{x}=0.05 \mathrm{~m}$

Fig. 6 Surface temperature evolution - comparing COMSOL and PIRATE 1D/Q2D for C/C uncooled.

gradient imposed at the lateral boundary of the surface heat flux. As shown in Fig. 6b, the temperature distributions are highly sensitive and a suitable $\mathrm{dx}$ needs to be picked for a case by case basis. Assuming $\mathrm{dt}=0.05 \mathrm{~s}$, for the subsequent simulations $\mathrm{dx}=0.0025 \mathrm{~m}$ is chosen which represents a peak temperature difference of $1.14 \%$ (or $4.59 \mathrm{~K}$ ). The simulation time for this fully 2D simulation is $11196 \mathrm{~s}$, around 3 hours (Table 3). It should be noted that a very fine spatial discretisation is required for this validation case, due to the large thermal gradients imposed. It is envisaged that for more uniform spatial heat flux distributions, sufficient accuracy can be achieved with a coarser mesh.

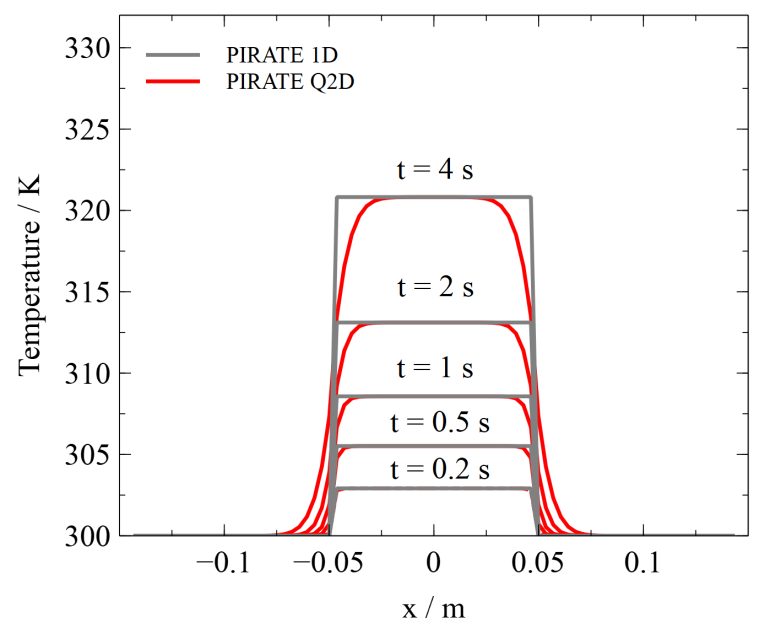

Fig. 7 Surface temperature evolution along the $x$ axis $-\mathrm{C} / \mathrm{C}$ uncooled.

Figure 7 shows the evolution of the surface temperature over several time steps for the $\mathrm{C} / \mathrm{C}$ case. Comparing the 
PIRATE 1D to the PIRATE Q2D code, it is clear that at later time steps more heat flux dissipates towards the sides, leading to a 'smoothing' of the surface temperature profile along the $\mathrm{x}$ axis. This demonstrates that PIRATE Q2D correctly models the transient lateral distribution of surface temperature.

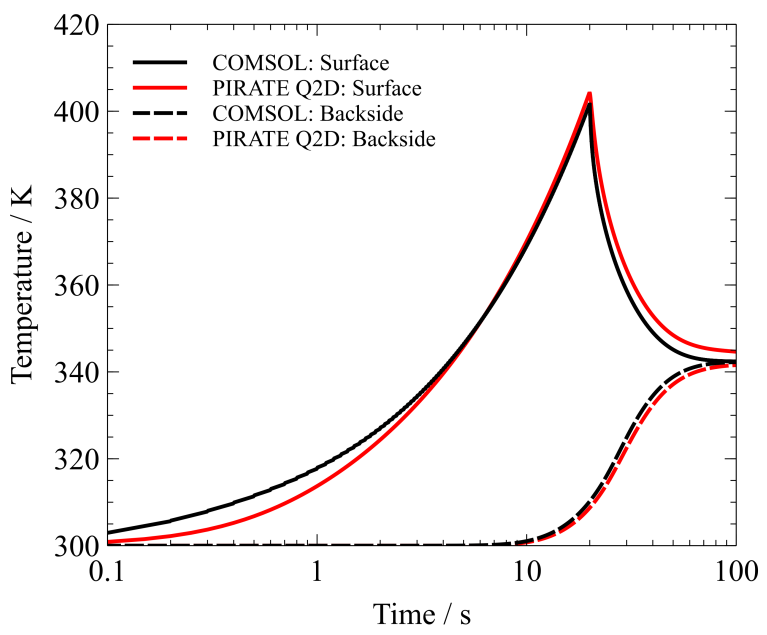

(a) $\mathrm{C} / \mathrm{C}$

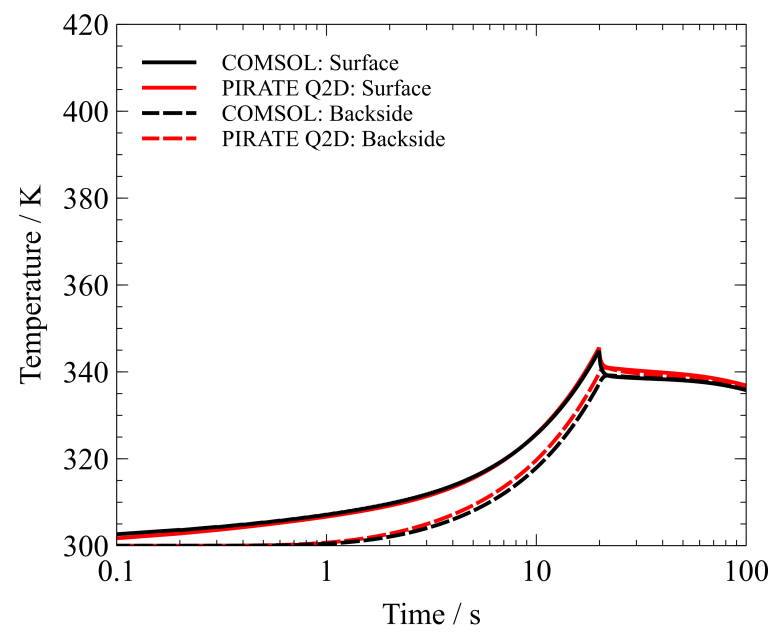

(b) UHTC

Fig. 8 Transient temperature evolution at $\mathbf{x}=\mathbf{0 . 0 5} \mathrm{m}$ - uncooled.

Figure 8 shows the transient surface and backside temperatures at the point $\mathrm{x}=0.05 \mathrm{~m}$ for both materials. This point represents the intersection between the heat flux input boundary condition and the adiabatic boundary around the model and is where considerable two-dimensional effects and the largest errors are expected. For the entire time history, the PIRATE Q2D simulations agree within 5\% of the COMSOL simulations, capturing the linear rise during the onset of heating and then the drop off as heat dissipates to rest of the material. For the C/C case, PIRATE Q2D under-predicts the temperature peak by $6 \mathrm{~K}$ which can be attributed to the time discretisation error described before. In addition, there is a slight over prediction of $4 \mathrm{~K}$ of the final temperature. This is consistent with both materials and is likely to be due to a lateral discretisation error $(\mathrm{dx}=0.01 \mathrm{~m}$ for these simulations $)$. As this point constitutes a very transient and non-linear change in temperature, a very small change in lateral discretisation leads to a large change in temperature.

Figure 9, shows the temperature distribution along the body for $\mathrm{C} / \mathrm{C}$ and UHTC at time $10 \mathrm{~s}$. Reinforcing the results above, the general shape of the temperature distribution for PIRATE Q2D matches closely with COMSOL. For the $\mathrm{C} / \mathrm{C}$ case, the difference of $12 \mathrm{~K}$ in peak surface temperature at $\mathrm{t}=10 \mathrm{~s}$ can again be attributed to the time discretisation error described before whilst the difference for UHTC is only $2.5 \mathrm{~K}$. Overall, the implementation of the quasi- two-dimensional correction appears to be of suitable accuracy for systems level calculations. 


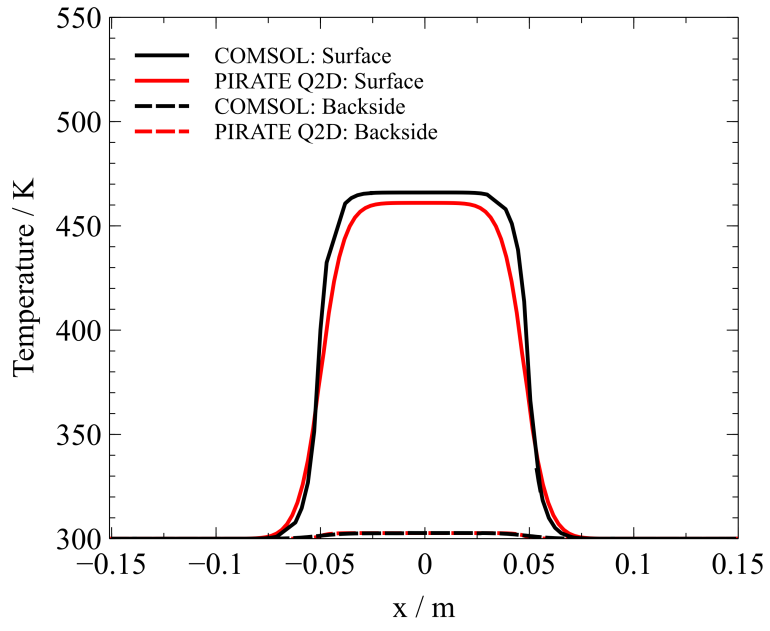

(a) $\mathrm{C} / \mathrm{C}$

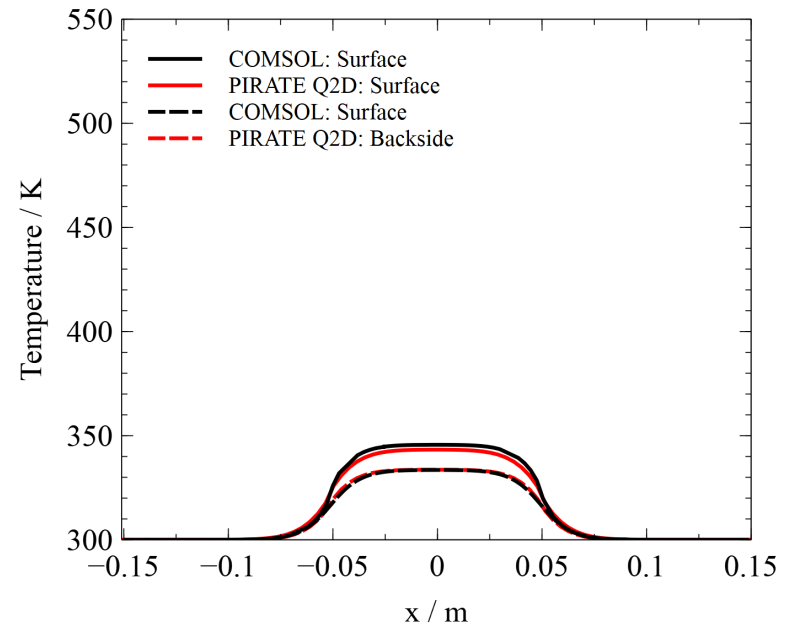

(b) UHTC

Fig. 9 Temperature distribution along the body at $t=10 \mathrm{~s}-$ uncooled.

\section{B. Cooled - SHEFEX II flight data}

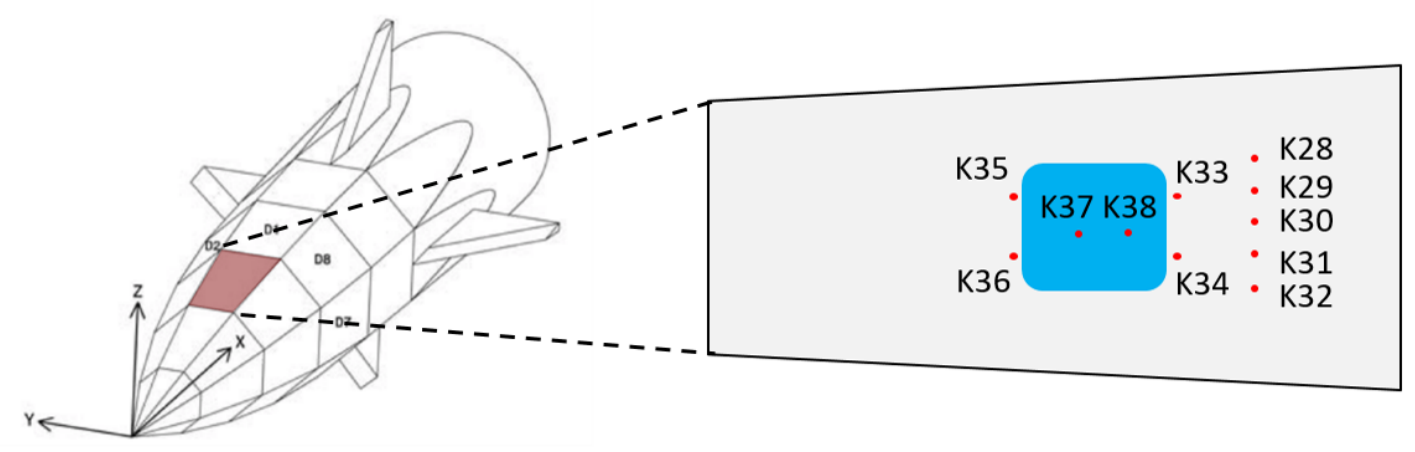

Fig. 10 AKTiV thermocouple locations on experiment - panel C3 [13].

To validate the lateral conduction model with transpiration cooling, the PIRATE code with lateral conduction has been applied to the SHEFEX flight data [13]. The total flight time was roughly $500 \mathrm{~s}$ with $52 \mathrm{~s}$ of experimental time for the atmospheric re-entry at altitudes between 100 and $30 \mathrm{~km}$. The SHEFEX II flight experiment implemented a transpiration-cooled experiment named AKTiV. Porous C/C samples (properties from Table 1 with a thickness of $5 \mathrm{~mm}$ were inserted in the centre of a $7 \mathrm{~mm}$ thick thermal protection panel made of $\mathrm{C} / \mathrm{C}-\mathrm{SiC}$ [13]. Nitrogen coolant gas was fed through the porous sample at a mass flow rate of $0.4 \mathrm{~g} / \mathrm{s}$ with a stainless steel plenum. Fig. 10 shows the locations of thermocouples for the AKTiV experiment at Panel C3. The reservoir temperature is assumed to be 300 K [13]. Before the cooling is started at $431 \mathrm{~s}$, the pre-heating of the model has been simulated by starting from the initial temperature distribution. 


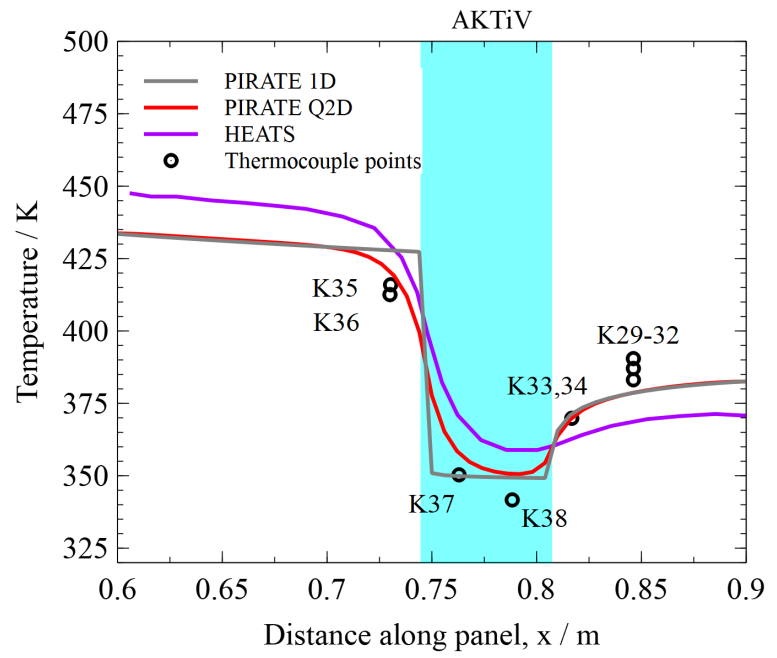

Fig. 11 Comparison of experimental to simulated temperatures along panel $\mathrm{C} 3$ at the surface of AKTiV at $\mathrm{t}=\mathbf{4 8 5 . 1 2}$ s [13].

The temperature distribution for the AKTiV flight experiment is shown in Fig.11 for $\mathrm{t}=485.12 \mathrm{~s}$ where the experimental results from the SHEFEX flight data, the two-dimensional finite difference HEATS simulation data, the PIRATE 1D and PIRATE Q2D simulations are plotted. The results show very good agreement within $2 \mathrm{~K}$ between PIRATE Q2D and the measured thermocouple data upstream of the porous sample (K35 \& K36). The HEATS two-dimensional finite difference code follows a very similar trend to PIRATE Q2D. The over prediction of HEATS may be attributed to fact that PIRATE 1D and Q2D account for the radiation exchange between the backside and the plenum whilst HEATS considers an adiabatic backside. The prediction of the temperature reduction due to cooling is in good accordance with both HEATS and the experimental data and lies within a margin of less than $10 \mathrm{~K}$. There is a slightly different trend in the downstream cooling between HEATS and both PIRATE simulations. This may be attributed to the different models used between HEATS and PIRATE to calculate the downstream film effectiveness. Where HEATS uses a boundary layer heat balance, PIRATE uses a semi-empirical correlation [8]. Both PIRATE models follow the surface thermocouple data closely, within $10 \mathrm{~K}$ for K29-32. Finally, a comparison between the PIRATE 1D and PIRATE Q2D simulations illustrate the increased accuracy attained by considering $2 \mathrm{D}$ effects, particularly at the boundary between the thermal protection outer panel and the porous sample. This gives confidence in the implementation of PIRATE Q2D with transpiration cooling.

\section{Coupled Numerical Code}

One of the key assumptions made by the PIRATE solver is that the boundary condition at the backside of the porous wall is represented as a radiative exchange between the back surface and a cold substructure (Fig. 2): 


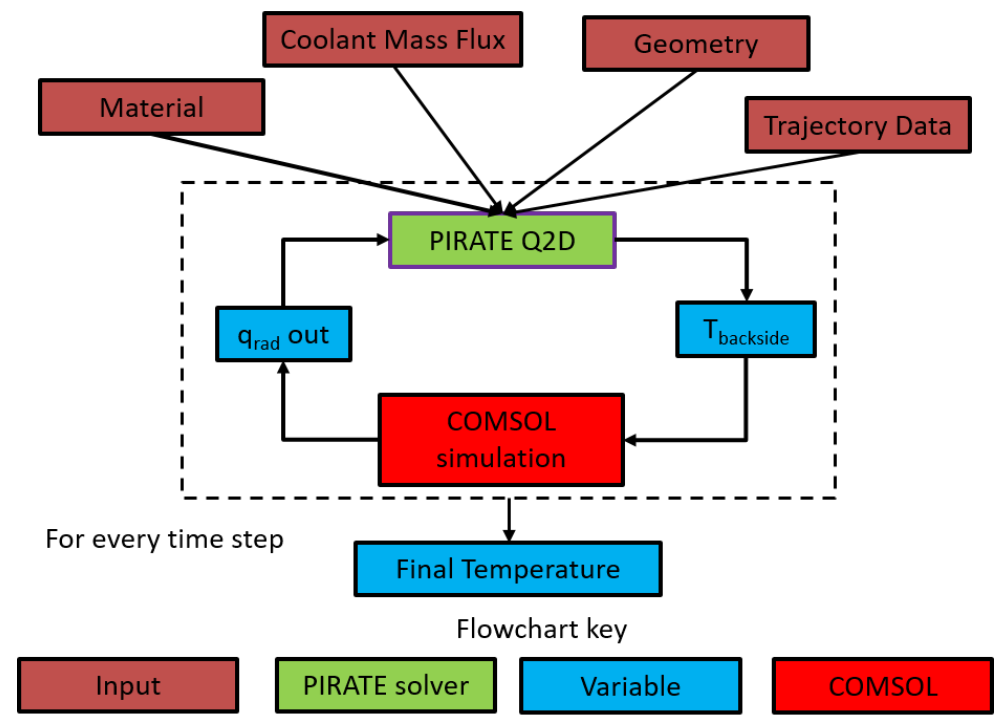

Fig. 12 Flowchart of coupled numerical model.

$$
\dot{q}_{\text {backside }}=-\frac{\sigma\left(T_{\text {backside }}^{4}-T_{\text {sub }}^{4}\right)}{\frac{1}{\epsilon_{s}}+\frac{1}{\epsilon_{\text {sub }}}-1}
$$

where $\sigma$ is the Boltzmann constant, $\epsilon_{\text {sub }}$ is the emissivity of the substructure and $\epsilon_{s}$ is the emissivity of the porous outer wall. In reality, during a flight trajectory, the substructure temperature $\left(T_{\text {sub }}\right)$ increases due to the incoming radiative heat flux from the backside of the porous wall. An increase in substructure temperature then leads to a reduction in the net negative backside heat flux which reduces the transient heat loss from the porous backside as the substructure heats up. Even if critical material temperature limits are maintained for the outer wall, overall failure for the vehicle is possible if the substructure temperature exceeds critical material limits.

To model this effect, the PIRATE Q2D code has been linked to the COMSOL finite element package using LiveLink for MATLAB. Figure 12 outlines a flowchart of the computational model. For each time step, the porous outer wall backside temperature calculated by PIRATE Q2D is taken as an input into a backside material boundary condition in COMSOL. Thereafter, once COMSOL calculates the heat transfer to the substructure for the given time step, the backside radiative heat flux is then taken as an input boundary condition for the porous wall backside in PIRATE Q2D. Henceforth, the coupled version of PIRATE Q2D with substructure is referred to as 'PIRATE Q2D+S.

A schematic of this model is shown for a generalised geometry in Fig. 13 where the entirety of the outer surface material (whether porous or solid) is modelled by PIRATE Q2D. For the substructure, an adiabatic boundary condition is assumed for the backside and a symmetric boundary condition along the stagnation point. For a given flight trajectory, the outer wall heat flux distribution is an input boundary condition calculated either using empirical correlations [8] or via an external database (e.g. from CFD).

The coupled numerical model presented assumes negligible convective heat transfer between the coolant channel 


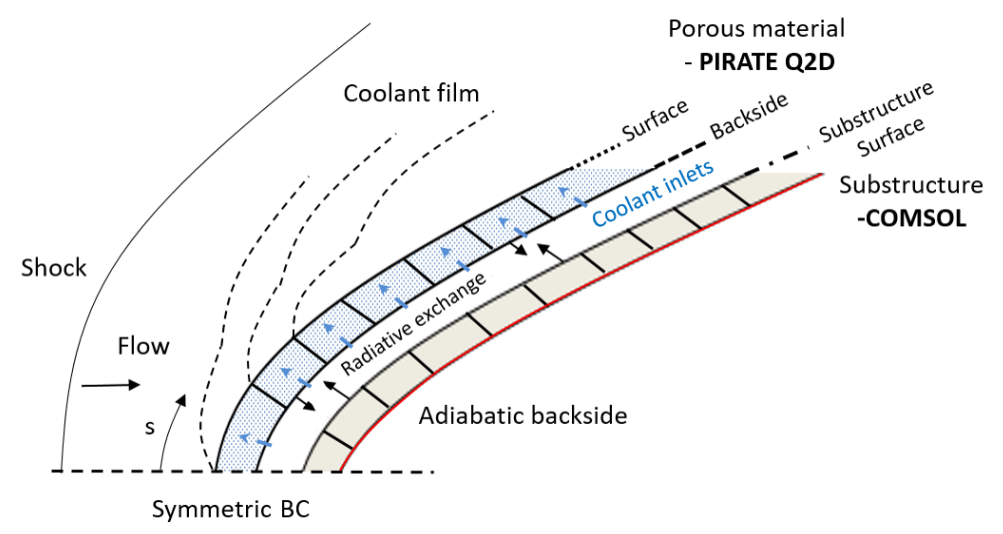

Fig. 13 Schematic of the two-dimensional model with substructure.

and the substructure. In addition the input coolant temperature at the backside of the porous surface is also assumed to at $300 \mathrm{~K}$. It is envisaged the coolant is be supplied to appropriate regions via channels through the substructure.

\section{Transient flight analysis with substructure}

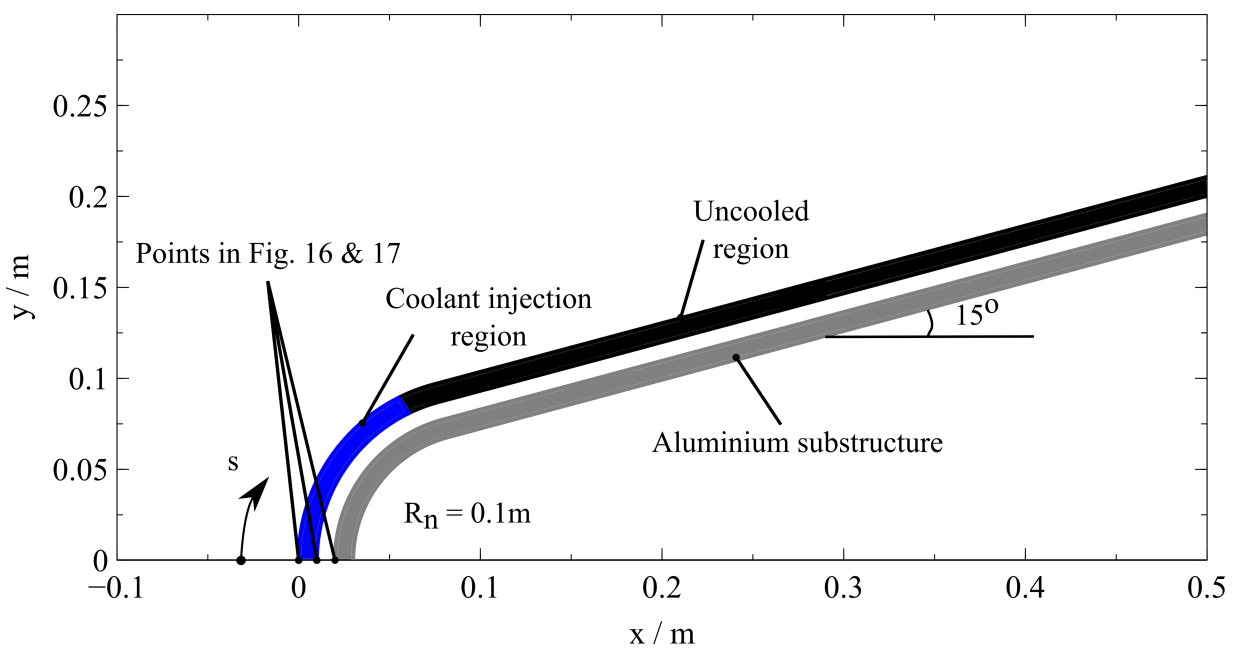

Fig. 14 Leading edge geometry considered in this study. Coolant injection region from $\mathrm{s}=0-0.11 \mathrm{~m}$.

To demonstrate an application of the coupled numerical approach detailed above, the study presented in this paper consists of a $15^{\circ}$ half angle wing leading edge with a $0.1 \mathrm{~m}$ nose radius and a consistent wall thickness of $\mathrm{L}=10 \mathrm{~mm}$. A simplified $10 \mathrm{~mm}$ aluminium thick substructure is considered with a gap of $10 \mathrm{~mm}$ between the porous wall and the substructure acting as a coolant reservoir. The total length from the stagnation point along the body profile is $0.57 \mathrm{~m}$ and a schematic of the model is displayed in Fig.14

The external heat flux boundary condition is determined by applying the Sutton Graves stagnation point correlation corrected for a 2D stagnation line [20]. Laminar flow is assumed at the external surface and the Lees laminar heat transfer correlation is applied for the varying heat flux boundary condition at the external surface [21]. 
For the porous outer wall, the $\mathrm{C} / \mathrm{C}$ and UHTC materials are chosen of which the material parameters are summarised in Table 1 The aluminium substructure is built up with assumed nominal properties of: emissivity, $\epsilon_{\text {sub }}=0.07$; density, $\rho=2700 \mathrm{~kg} \mathrm{~m}^{-3}$; specific heat capacity, $c_{p}=900 \mathrm{~J} \mathrm{~kg}^{-1} \mathrm{~K}^{-1}$ and thermal conductivity, $k=177 \mathrm{~W} \mathrm{~m}^{-1} \mathrm{~K}^{-1}$. Heat transfer through any supports between the substructure and the porous outer wall are neglected as well as any heat transfer between the coolant gas and the substructure. This assumption is sensible as any heat transfer through the supports are likely to be very small if they are made of highly insulating materials [13]. In addition, the primary purpose of this study is to demonstrate the purely radiative heat transfer interplay between the porous wall and the substructure. In addition, it is assumed that there is no radiative shielding and that there are no coatings to reduce the emissivity at the backside of the porous wall which would be present in a real spacecraft.

As per Kays et al. [10], a commonly used parameter to describe transpiration cooled processes is the ratio of coolant transpired through the wall and the main flow, also known as the blowing ratio defined as:

$$
F=\frac{\rho_{f} u_{f}}{\rho_{g} u_{g}}=\frac{\dot{m}_{A}}{\rho_{g} u_{g}}
$$

with coolant mass flux, $\dot{m}_{A}\left(\mathrm{~kg} \mathrm{~s}^{-1} \mathrm{~m}^{-2}\right)$, where (g) and (f) denote values for the post shock gas and coolant respectively. Further details regarding the calculation of heat fluxes and blowing ratios from the free-stream conditions are detailed in Ref. [8].

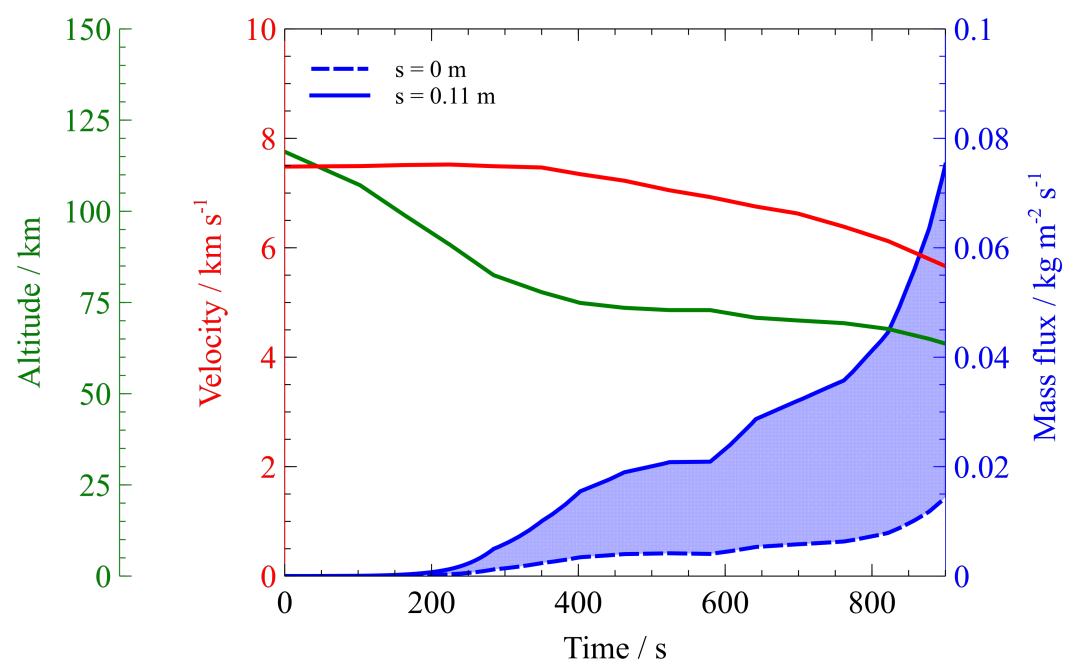

Fig. 15 Space Shuttle re-entry trajectory with the coolant mass flux distribution within the coolant injection region, $F=0.04$

The following simulations are all carried out for the first 900 s of the Space Shuttle re-entry trajectory [22] where the model is assumed to be at a uniform initial temperature of $300 \mathrm{~K}$. Transpiration cooling is applied by injecting Helium coolant at $300 \mathrm{~K}$ at four constant blowing ratios, $F$, of: $0.01,0.02,0.03$ and 0.04 . Figure 14 shows the coolant injection 
region from $\mathrm{s}=0$ to $0.11 \mathrm{~m}$. This has been chosen based on the conclusions in Ref. [17] which showed for a number of trajectories that transpiration cooling is only needed near the nose to maintain a vehicle below critical temperature limits. The mass flux distribution at the coolant injection region is shaded in Fig. 15 over the course of the trajectory for a constant blowing ratio, $F=0.04$. As expected at later points, to maintain a constant blowing ratio, the coolant injection mass fluxes increase greatly due to the higher free-stream gas density at lower altitudes. The boundary layer is assumed to be laminar for the duration of the chosen trajectory. This assumption is justified by considering Ref. [17] which showed, through a survey of different transpiration cooling experiments, that the boundary layer is not tripped if the blowing ratio is kept below 0.04 .

Each PIRATE Q2D+S simulation requires between 9-11 hours to complete. The computation time is significantly slower compared to the PIRATE Q2D simulations detailed in Section IV mainly due to the coupling process between PIRATE and COMSOL. However, this is still well within a reasonable time frame for a complex transient conjugate heat transfer simulation and highly applicable for large scale systems studies.

\section{A. Uncooled coupled numerical simulation}

Firstly, the PIRATE Q2D+S code is compared to PIRATE Q2D and PIRATE 1D for the uncooled case (zero coolant injection). As the PIRATE 1D and Q2D model assumes that the substructure has infinite thermal capacity, this remains at a temperature of $300 \mathrm{~K}$ throughout the flight trajectory. PIRATE Q2D+S transiently calculates the change in substructure temperature due to the incoming radiative heat flux from the backside of the porous wall.

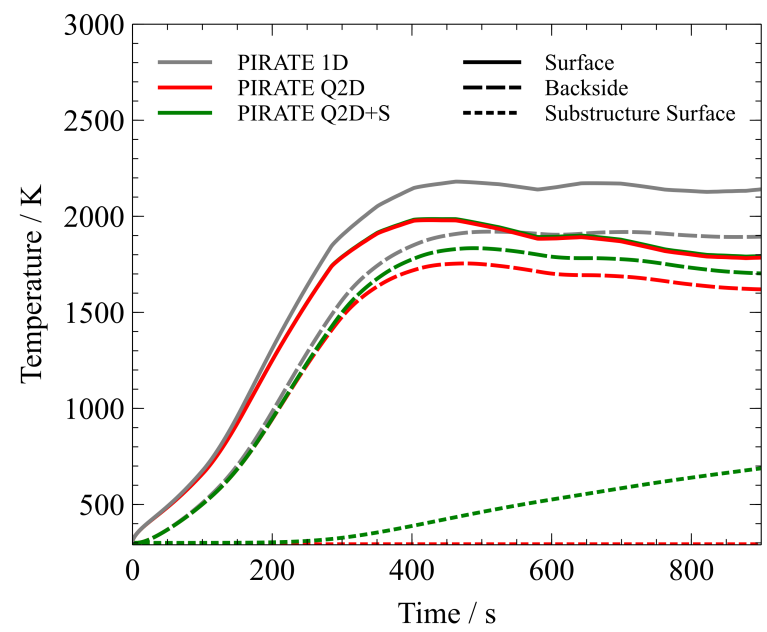

(a) $\mathrm{C} / \mathrm{C}$

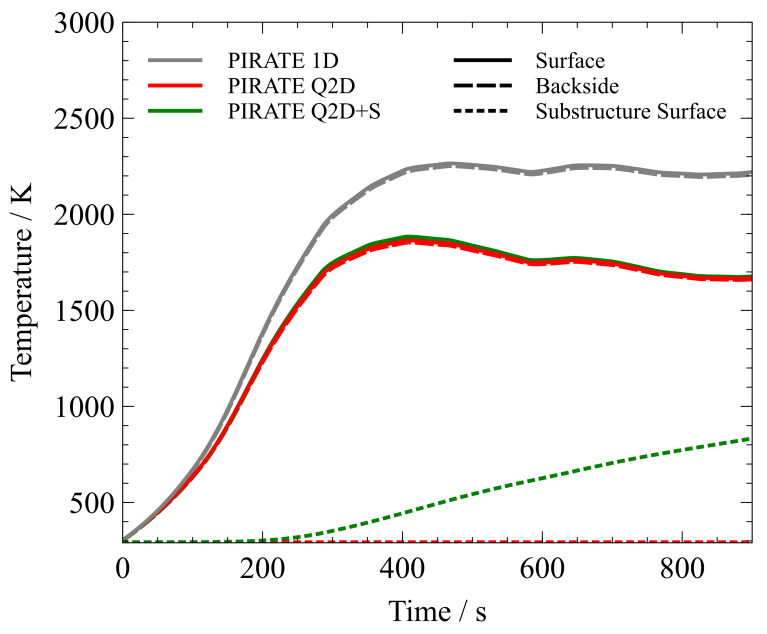

(b) UHTC

Fig. 16 Transient temperature evolution at the stagnation point ( $\mathrm{s}=0 \mathrm{~m})$, comparison of PIRATE 1D, Q2D and Q2D+S uncooled. 
Figure 16 shows the transient temperature history of the surface and backside of the $\mathrm{C} / \mathrm{C}$ and UHTC porous wall and the surface temperature of the substructure at the stagnation point. By first comparing PIRATE 1D and 2D, as expected, the $1 \mathrm{D}$ simulation significantly overestimates the temperature rise over the trajectory, diverging by $340 \mathrm{~K}$ at the final time step. This is due to the large thermal gradients near the stagnation point for the wing leading edge geometry and justifies the development and application of the quasi two-dimensional method to account for the significant lateral conduction effects. The lower material emissivity of UHTC results in reduced radiative cooling. This is balanced out by the significantly lower heat capacity which leads to largely similar surface temperatures between UHTC and C/C for the one-dimensional simulation ( $2250 \mathrm{~K}$ vs $2180 \mathrm{~K}$ at the maximum point for PIRATE 1D). However, the appreciably higher thermal conductivity of UHTC means that heat is very quickly conducted away from the stagnation point and results in a lower two-dimensional peak temperature.

Considering PIRATE Q2D+S, there is a significant temperature increase of the substructure over the trajectory, reaching a peak of $700 \mathrm{~K}$ for $\mathrm{C} / \mathrm{C}$. The steady, linear rise in temperature is plausible as the backside absorbs the total integrated heat flux. The back face of the substructure is assumed to be adiabatic with no further heat transfer into internal structures. In addition, as the aluminium surface temperature increases, there is greater radiation emission leading to a net backside heat flux which raises the backside temperature. As the emissivity of aluminium is very low, the temperature rise is only a maximum of 70-80 K. For UHTC, the higher thermal conductivity leads to an equilibration between the surface and backside temperatures over the trajectory. The corresponding higher backside heat flux results in a maximum temperature of $850 \mathrm{~K}$ at the substructure surface illustrating that UHTC acts as a hot structure in this trajectory, dissipating heat quickly to the underlying body.

\section{B. Application of cooling}

Secondly, transpiration cooling is applied within the injection region for the four different constant blowing ratios detailed previously. Figure 17 illustrates the effect of gas injection to the stagnation region for the $\mathrm{C} / \mathrm{C}$ and UHTC materials where $F=0.04$ of Helium coolant is injected. For $\mathrm{C} / \mathrm{C}$, as expected, this leads to a reduction in both the surface and backside temperatures of the porous wall. The reduction in backside temperature then results in a lower heat flux from radiative emission at the substructure and thereby a significantly lower substructure temperature rise. As the backside temperatures are reduced along the entire trajectory, a moderate reduction in the peak backside temperature of the outer wall (approximately $180 \mathrm{~K}$ ) leads to a $250 \mathrm{~K}$ reduction in the substructure temperature at the final trajectory point to $440 \mathrm{~K}$, a $36 \%$ drop compared to the uncooled case. The backside and surface temperatures diverge at later times as to maintain the same blowing ratio requires a greater coolant mass flux, and thus there is increased internal convective cooling. This effect shows that in addition to the use of transpiration cooling to reduce outer wall surface temperatures, there is an advantageous side effect where the integrated heat loads to the substructure can also be lowered when coolant is injected for a large portion of the trajectory. For the UHTC material, at the application of cooling, the 


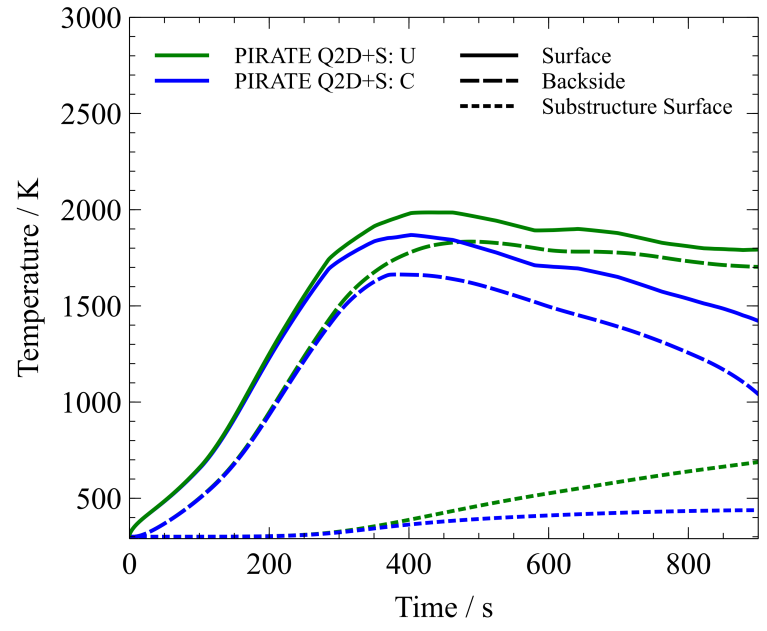

(a) $\mathrm{C} / \mathrm{C}$

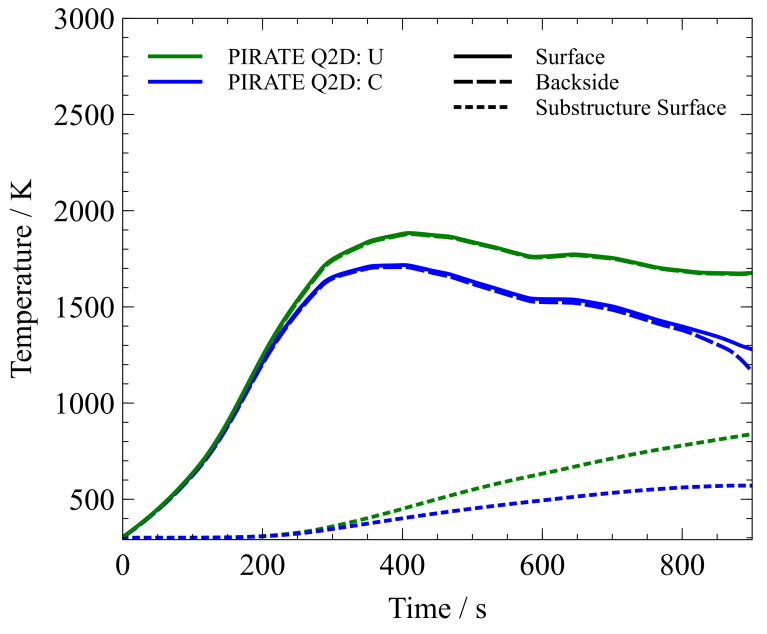

(b) UHTC

Fig. 17 Influence of coolant injection at the stagnation point. $\mathrm{U}=$ uncooled, $\mathrm{C}=$ cooled with $F=0.04$ of Helium coolant injection.

final temperature at the stagnation point drops by only $50 \mathrm{~K}$ to $880 \mathrm{~K}$. The lower temperature reduction compared to $\mathrm{C} / \mathrm{C}$ is expected primarily due to the higher backside temperatures of UHTC, caused by the high thermal conductivity of UHTC. Therefore, transpiration cooling has higher benefits for the substructure when heat conduction is slow in the material.

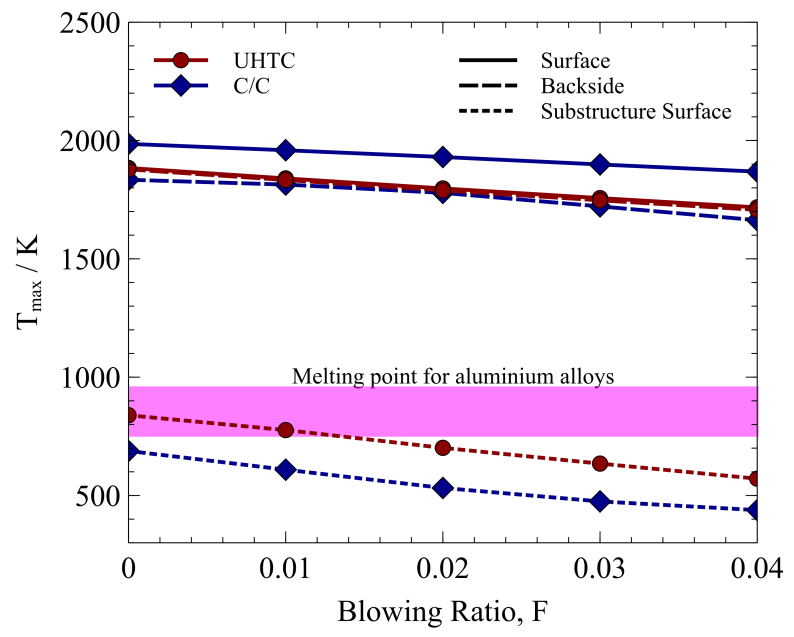

Fig. 18 Maximum temperature for various blowing ratios. The location of these temperature peaks is at the stagnation point $(\mathrm{s}=0 \mathrm{~m})$.

Figure 18 shows how the maximum temperatures of the surface, backside and substructure reduce with increasing blowing ratios. For the surface, both C/C and UHTC show an approximately linear trend where increasing the blowing 
ratio from 0 to 0.04 reduces maximum temperatures by 125 and $160 \mathrm{~K}$ respectively for $\mathrm{C} / \mathrm{C}$ and UHTC. The substructure temperatures reduce at a greater rate (approximately $250 \mathrm{~K}$ for $\mathrm{C} / \mathrm{C}$ and $165 \mathrm{~K}$ for UHTC) as this represents the integrated load over the entire trajectory. Between $F=0.03$ and 0.04 , there is a slowdown in the reduction of substructure temperatures ratios; suggesting a reduced marginal benefit with higher blowing ratios. By considering the melting point of various aluminium alloys, it is clear that for this particular trajectory and geometry, even a moderate level of coolant injection is highly beneficial for UHTC and can reduce the substructure temperature below critical limits.

\section{Impact on thermal gradients}

The extreme thermal gradients present lead to large thermal stresses near the stagnation point of hypersonic wing leading edges. These are a significant engineering challenge and can frequently violate structural limits. This is important for both the outer wall and the underlying substructure. Here, a baseline study is conducted on the impact of transpiration cooling to the substructure thermal gradients.

The thermal expansion of a material is proportional to its temperature gradient; therefore a variation in temperature, $\Delta T$, across a material induces a thermal stress $\sigma_{\text {th }}$, given by:

$$
\sigma_{\text {th }} \propto \Delta T
$$

To quantify this benefit, $\Delta T_{\text {sub,lateral }}$ is defined as the change in substructure surface temperature between the stagnation point and at $\mathrm{s}=0.56 \mathrm{~m}$ :

$$
\Delta T_{\text {sub,lateral }}=T_{\text {sub }, \mathrm{s}=0 \mathrm{~m}}-T_{\text {sub }, \mathrm{s}=0.56 \mathrm{~m}}
$$

where it is assumed that the substructure is fixed at these two locations so that any temperature gradient between these points directly corresponds to an increased thermal stress. Focusing on the C/C material only, Fig. 19a shows

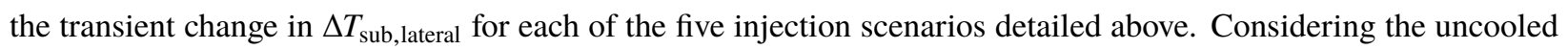
case, as expected, there is very little change for the first $200 \mathrm{~s}$ as the heat flux slowly propagates from the surface to the substructure. Thereafter there is a rapid increase peaking at $\Delta T_{\text {sub,lateral }}=225 \mathrm{~K}$. For coolant injection, the maximum level of cooling of $\mathrm{F}=0.04$ leads to a maximum $\Delta T_{\text {sub,lateral }}$ of $80 \mathrm{~K}$, a $65 \%$ reduction. As $\Delta T_{\text {sub,lateral }}$ is directly proportional to the thermal stress, transpiration cooling can be very effective in reducing the high thermal stresses near the stagnation region of the leading edge.

Figure $19 \mathrm{~b}$ shows the substructure temperature distribution along the body at $\mathrm{t}=900 \mathrm{~s}$. The effect of coolant injection is significantly reducing the temperature gradient near the stagnation point of the leading edge. Even though the coolant is only applied close to the stagnation region, temperatures also reduce significantly far downstream with a $65 \mathrm{~K}$ reduction at $\mathrm{s}=0.56 \mathrm{~m}$ between the uncooled case and a blowing ratio of 0.04 . This is primarily due to the high 


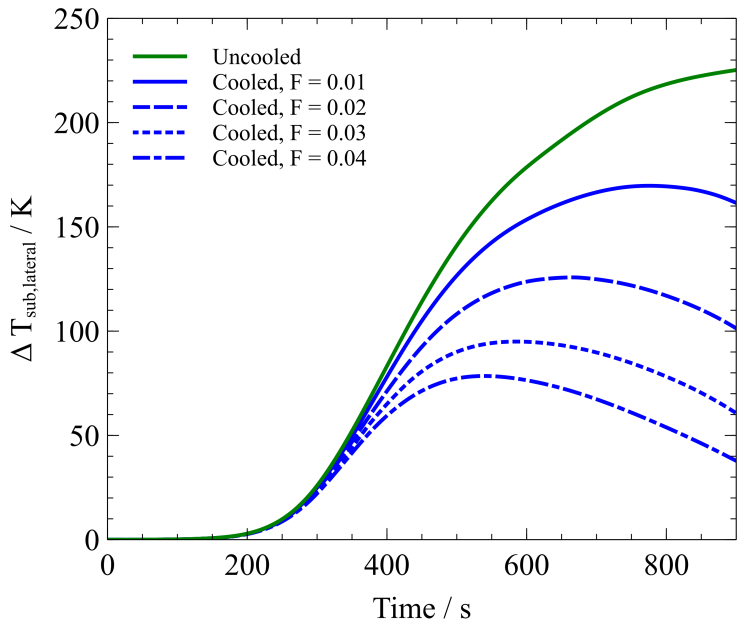

(a) Transient change in $\Delta T_{\text {sub,lateral }}$

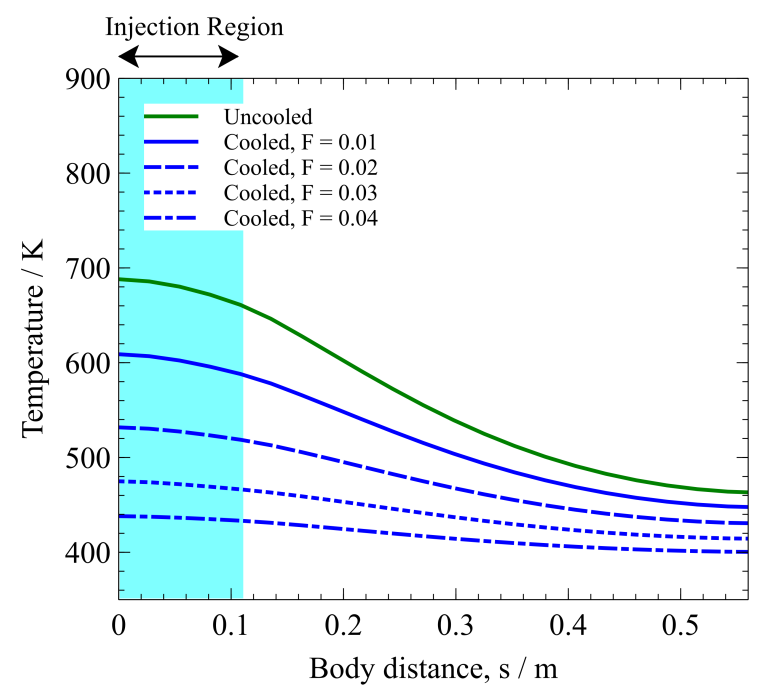

(b) Temperature distribution along the body, $t=900 \mathrm{~s}$

Fig. 19 An assessment of the temperature gradient along the substructure surface at the onset of cooling - C/C outer porous wall.

conductivity of aluminium which quickly conducts heat away from the stagnation point to locations further downstream. In addition, the propagation of the coolant film downstream of the injection region leads to a moderate reduction in the surface temperature and hence a reduction in substructure temperature .

\section{Conclusions}

This paper presents improvements to the PIRATE code developed to perform rapid simulations of the transient temperature evolution of transpiration cooled heat shields for hypersonic vehicles. The Crank-Nicholson numerical scheme has been applied to the existing PIRATE code, providing the capability to accurately model lateral conduction for geometries with moderate thermal gradients and strictly one dimensional coolant mass flow. The quasi two-dimensional method allows for rapid calculations of the transient temperature history, suitable for large scale systems studies with minimal computational cost. The model is validated against COMSOL and experimental data from the SHEFEX II re-entry flight test. An uncooled validation case using COMSOL showed excellent agreement with the quasi two-dimensional method, accurately capturing the transient non-linear temperature profile. The comparison between simulation results and the SHEFEX II re-entry flight test experimental data showed good agreement within $10 \mathrm{~K}$, demonstrating that the lateral conduction effects at the onset of transpiration cooling are being resolved.

To model the substructure of a vehicle where transpiration cooling is applied, the PIRATE code is coupled to COMSOL to create a tool by which complete systems studies can be investigated. Preliminary results show the heating up of the substructure is a major factor in choosing appropriate materials for a given mission trajectory. Applying transpiration cooling has the effect of not only reducing temperatures for the outer wall but also significantly reducing 
the temperature of the substructure. By applying a moderate level of coolant injection $(F=0.04$ of Helium $)$, the maximum temperature of the substructure surface reduces from over $700 \mathrm{~K}$ to $450 \mathrm{~K}$. Furthermore, transpiration cooling can reduce the thermal stresses at the substructure, with a $65 \%$ reduction in the temperature gradient of the substructure surface for moderate coolant injection.

For the purposes of this paper, a generalised wing leading edge geometry and a constant thickness substructure are considered. However, in principle, any heat flux distribution can be applied as an input from an external database (e.g. CFD) and a complex substructure built up within the COMSOL environment. In the future, it is envisaged that this tool will be developed to perform optimisation studies for a fully formed and realistic transpiration cooling system.

\section{Acknowledgements}

This research is funded by the EPSRC grant "Transpiration Cooling Systems for Jet Engine Turbines and Hypersonic Flight" (reference: EP/P000878/1). The authors of this paper would like to acknowledge James Barth and Gerrie Mullen for their input in the initial stages of this study. The authors would also like to thank the Reaction Engines \& EPSRC CASE reward for supporting the studentship of Imran Naved. The authors would also like to extend their gratitude toward the reviewers who improved the quality of this paper.

\section{References}

[1] Schweikert, S., and Hald, H., "Characterization of Actively Cooled Porous C/C Wall Segments According to Pressure Loss and Internal Temperature Distribution,” Tech. rep., 2013. URL https://elib.dlr.de/86614/

[2] Rocher, M. E., Hermann, T., McGilvray, M., Ifti, H. S., Hufgard, F., Eberhart, M., Meindl, A., Löhle, S., Giovannini, T., and Vandeperre, L., "Testing a transpiration cooled zirconium-di-boride sample in the plasma tunnel at irs," AIAA Scitech 2019 Forum, American Institute of Aeronautics and Astronautics Inc, AIAA, 2019. doi:10.2514/6.2019-1552, URL https://arc.aiaa.org/doi/abs/10.2514/6.2019-1552

[3] Colwell, G. T., and Modlin, J. M., "Heat pipe and surface mass transfer cooling of hypersonic vehicle structures," Journal of Thermophysics and Heat Transfer, Vol. 6, No. 3, 1992, pp. 492-499. doi:10.2514/3.387, URL https://arc.aiaa.org/doi/abs/10. $2514 / 3.387$

[4] Modlin, J. M., and Colwell, G. T., "Surface cooling of scramjet engine inlets using heat pipe, transpiration, and film cooling," Journal of Thermophysics and Heat Transfer, Vol. 6, No. 3, 1992, pp. 500-504. doi:10.2514/3.388, URL https://arc.aiaa.org/doi/10.2514/3.388

[5] Edney, B., "Anomalous Heat Transfer and Pressure Distributions on Blunt Bodies at Hypersonic Speeds in the Presence of an Impinging Shock.” Tech. rep., 1968. URL https://www.osti.gov/biblio/4480948-anomalous-heat-transfer-pressure-distributionsblunt-bodies-hypersonic-speeds-presence-impinging-shock 
[6] Edney, B. E., "Effects of shock impingement on the heat transfer around blunt bodies," AIAA Journal, Vol. 6, No. 1, 1968, pp. 15-21. doi:10.2514/3.4435, URL https://arc.aiaa.org/doi/10.2514/3.4435

[7] Boehrk, H., Piol, O., and Kuhn, M., "Heat Balance of a Transpiration-Cooled Heat Shield," Journal of Thermophysics and Heat Transfer, Vol. 24, No. 3, 2010, pp. 581-588. doi:10.2514/1.47172, URL https://arc.aiaa.org/doi/10.2514/1.47172

[8] Hermann, T., Naved, I., and McGilvray, M., “Tool for Rapid Transient Transpiration-Cooled Reentry Simulation,” AIAA Journal, Vol. 58, No. 2, 2020, pp. 842-853. doi:10.2514/1.j058516, URL/https://arc.aiaa.org/doi/10.2514/1.J058516

[9] Steelant, J., Dalenbring, M., and Wang, G., “The Thermal and Structural Paradox for Hypersonic Cruisers," Proceedings of the 8th European Symposium on Aerothermodynamics for Space Vehicles, , No. 1, 2015.

[10] Kays, W. M., Crawford, M. E., and Weigand, B., Convective Heat and Mass Transfer, $4^{\text {th }}$ ed., McGraw-Hill, New York, 2005.

[11] Volchkov, E. P., Zaulichnyi, E. G., Kutateladze, S. S., and Leont'ev, A. I., "Film cooling by injection into a turbulent boundary layer," Journal of Applied Mechanics and Technical Physics, Vol. 8, No. 2, 1967, pp. 63-64. doi:10.1007/BF00918038, URL https://link.springer.com/article/10.1007/BF00918038

[12] Glass, D. E., Dilley, A. D., and Kelly, H. N., "Numerical Analysis of Convection / Transpiration Cooling," Journal of Spacecraft and Rockets, Vol. 38, No. 1, 2008, pp. 15-20. doi:10.2514/2.3666, URL https://arc.aiaa.org/doi/10.2514/2.3666

[13] Böhrk, H., “Transpiration-Cooled Hypersonic Flight Experiment: Setup, Flight Measurement, and Reconstruction,” Journal of Spacecraft and Rockets, Vol. 52, No. 3, 2015, pp. 674-683. doi:10.2514/1.a33144, URL https://arc.aiaa.org/doi/10.2514/1. A33144

[14] Kuhn, M., and Hald, H., "Application of transpiration cooling for hot structures," Notes on Numerical Fluid Mechanics and Multidisciplinary Design, Vol. 98, 2008, pp. 82-103. doi:10.1007/978-3-540-77819-6_6.

[15] Hughes, T. J. R., "Unconditionally stable algorithms for nonlinear heat conduction,” Computer Methods in Applied Mechanics and Engineering, Vol. 10, No. 2, 1977, pp. 135-139. doi:https://doi.org/10.1016/0045-7825(77)90001-9, URL http://www. sciencedirect.com/science/article/pii/0045782577900019

[16] COMSOL AB, “COMSOL Multiphysics®,”, 2018. URL www.comsol.com

[17] Hermann, T., McGilvray, M., and Naved, I., "Performance of Transpiration-Cooled Heat Shields for Reentry Vehicles," AIAA Journal, Vol. 58, No. 2, 2020, pp. 830-841. doi:10.2514/1.J058515, URL https://arc.aiaa.org/doi/10.2514/1.J058515

[18] Ifti, H. S., Hermann, T., and McGilvray, M., "Flow characterisation of transpiring porous media for hypersonic vehicles," 22nd AIAA International Space Planes and Hypersonics Systems and Technologies Conference, American Institute of Aeronautics and Astronautics Inc, AIAA, 2018. doi:10.2514/6.2018-5167, URL https://arc.aiaa.org/doi/abs/10.2514/6.2018-5167

[19] Innocentini, M. D., Rizzi, A. C., Nascimento, L. A., and Pandolfelli, V. C., "The pressure-decay technique for air permeability evaluation of dense refractory ceramics," Cement and Concrete Research, Vol. 34, No. 2, 2004, pp. $293-298$. doi: 10.1016/j.cemconres.2003.08.006, URL https://www.sciencedirect.com/science/article/pii/S0008884603002849 
[20] Sutton, K., and Graves Jr, R. A., “A general stagnation-point convective heating equation for arbitrary gas mixtures,” 1971.

[21] Lees, L., "Laminar Heat Transfer Over Blunt-Nosed Bodies at Hypersonic Flight Speeds," Journal of Jet Propulsion, Vol. 26, No. 4, 2012, pp. 259-269. doi:10.2514/8.6977, URL https://arc.aiaa.org/doi/abs/10.2514/8.6977.

[22] Ko, L. W., Quinn, R. D., and Gong, L., “Finite-Element Reentry Heat-Transfer Analysis of Space Shuttle Orbiter,” Nasa technical paper 2657, NASA Ames Research Center, 1986. 